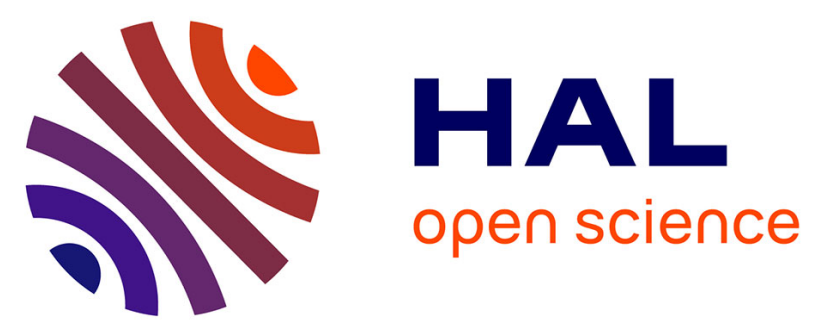

\title{
Qualification de l'efficacité du nettoyage du laveur désinfecteur à irrigation ultrasonique Medisafe SI PCF
}

A. Daguet, F. Benoit, V. Antoine, B. Demoré

\section{To cite this version:}

A. Daguet, F. Benoit, V. Antoine, B. Demoré. Qualification de l'efficacité du nettoyage du laveur désinfecteur à irrigation ultrasonique Medisafe SI PCF. Le Pharmacien Hospitalier et Clinicien, 2019, 54, pp.131 - 136. 10.1016/j.phclin.2018.12.002 . hal-03486270

\section{HAL Id: hal-03486270 \\ https://hal.science/hal-03486270}

Submitted on 20 Dec 2021

HAL is a multi-disciplinary open access archive for the deposit and dissemination of scientific research documents, whether they are published or not. The documents may come from teaching and research institutions in France or abroad, or from public or private research centers.
L'archive ouverte pluridisciplinaire HAL, est destinée au dépôt et à la diffusion de documents scientifiques de niveau recherche, publiés ou non, émanant des établissements d'enseignement et de recherche français ou étrangers, des laboratoires publics ou privés.

\section{(ㄷ)(1) $\$$}

Distributed under a Creative Commons Attribution - NonCommerciall 4.0 International 


\section{Qualification de l'efficacité du nettoyage du laveur désinfecteur à irrigation ultrasonique Medisafe SI PCF \\ Qualification of the efficiency of the ultrasonic cleaner Medisafe SI PCF}

\section{Auteur correspondant :}

Alexandra DAGUET, Interne en pharmacie, Hôpitaux de Brabois, Service PharmacieStérilisation, CHRU de Nancy, Rue du Morvan, 54511 VANDOEUVRE-LES-NANCY alexandra.daguet@gmail.com

06.19.91.79.94

Francis BENOIT, Pharmacien Praticien Hospitalier, Responsable du service de Stérilisation, Hôpitaux de Brabois, Service Pharmacie-Stérilisation, CHRU de Nancy, Rue du Morvan, 54511 VANDOEUVRE-LES-NANCY

Véronique ANTOINE, Cadre de santé, Hôpitaux de Brabois, Service PharmacieStérilisation, CHRU de Nancy, Rue du Morvan, 54511 VANDOEUVRE-LES-NANCY

Béatrice DEMORE, Pharmacien Praticien Hospitalier, Professeur de l'Université de Lorraine, Pharmacien Gérant, Chef de Pôle, Hôpitaux de Brabois, Service PharmacieStérilisation, CHRU de Nancy, Rue du Morvan, 54511 VANDOEUVRE-LES-NANCY

\section{Conflits d'intérêt : aucun.}




\section{Résumé}

Objectif. - Avant son utilisation au quotidien, l'équipe de stérilisation a souhaité contrôler l'efficacité du nettoyage de son nouveau bac à ultrasons Medisafe SI PCF.

Méthode. - Plusieurs types de pinces robot recouvertes de Soiltest ${ }^{\circledR}$, des tests de salissures STF Load Check® et Tosi-Lumcheck® ont été soumis au cycle Da Vinci, cycle spécifique aux pinces du robot Da Vinci, programmé par le fournisseur. En fin de cycle, le Résitest ${ }^{\circledR}$ a été employé afin de vérifier l'absence de protéines résiduelles. Des séries supplémentaires comportant uniquement les tests STF Load Check® et Tosi-Lumcheck® ont été effectuées selon le cycle standard, d'une durée plus courte sans phase de désinfection thermique.

Résultats. - Trente-deux pinces sur 119 ont été retrouvées sales après lavage (27\%), principalement les porte-aiguilles et les pinces fenêtrées. Le Résitest ${ }^{\circledR}$ n’a pas viré pour les pinces visuellement propres (100\%). Sur les 78 STF Load Check®de la charge, huit n'ont pas viré en cycle Da Vinci et un seul sur 35 n'a pas viré en cycle standard. Les Tosi-Lumcheck® ont tous été propres après lavage (56/56).

Conclusion. - Le taux de non-conformités a permis d'insister auprès du personnel qu'un brossage-écouvillonnage est essentiel. Un contrôle régulier du nettoyage par des tests de salissures est en cours de discussion.

Mots clés : Contrôle ; Nettoyage ; Qualification ; Stérilisation ; Ultrasons 


\section{$\underline{\text { Abstract }}$}

Objective. - Before its daily use, the sterilization team wanted to control the efficacy of the cleaning for their new Medisafe SI PCF ultrasonic cleaner.

Methodology. - Several types of robotic pliers tarnished with Soiltest ${ }^{\circ}$, STF Load Check® and Tosi-Lumcheck ${ }^{\circledR}$ soiling tests were submitted to Da Vinci cycle, a specific cycle for the Da Vinci robot's pliers, set by the supplier.At the end of the cycle, Resitest ${ }^{\circledR}$ was used to check for the absence of residual proteins. Additional series including only the STF Load Check ${ }^{\circledR}$ and Tosi-Lumcheck ${ }^{\circledR}$ tests were performed following a standard cycle (shorter duration and no thermal disinfection phase).

Results. - Thirty-two in 119 pliers remained dirty after washing (27\%), especially needle driver and fenestrated bipolar forceps. Resitest ${ }^{\circledR}$ didn’t turn for visually clean pliers (100\%). Of the 78 STF Load Check ${ }^{\circledR}$ distributed in the tray, eight did not turn after Da Vinci cycle and only one out in 35 did not turn after standard cycle. The Tosi-Lumcheck ${ }^{\circledR}$ were all clean after washing (56/56).

Conclusion. - The rate of non-compliance has made it clear to staff that brushing and cleaning with a bottle-brush is essential. Regular control of cleaning by soiling is under discussion.

Keywords: Control; Cleaning; Qualification; Sterilization; Ultrasound 


\section{Introduction}

Le service de stérilisation s'est doté début 2018 du nouveau laveur-désinfecteur à irrigation ultrasonique Medisafe SI PCF commercialisé par la société Amcor®. Il présente pour avantages, contrairement au bac à ultrasons utilisé précédemment dans le service (L 4407 BUS de Lupus Electro®), d'associer une irrigation ultrasonique, un nettoyage, un dégazage, une désinfection thermique et un rinçage final dans un même programme [1]. Avant cette acquisition, le matériel pris en charge était préalablement rincé, brossé et irrigué si nécessaire à l'aide d'une seringue remplie de détergent-désinfectant avant passage dans le bac à ultrasons, suivi d'un rinçage manuel à l'eau, extérieur et intérieur, à l'aide d'une seringue. Le matériel pris en charge en bac à ultrasons se compose des pinces du robot Da Vinci (irrigables), des coagulateurs à foie (irrigables), des alésoirs (irrigables), des clamps Cygnet® (irrigables) et des pinces urétéroscope (non irrigables).

Les qualifications d'usage (qualification d'installation, qualification opérationnelle et qualification de performances) du nouveau matériel ont été réalisées par le fournisseur à la mise en route de l'appareil pour les deux cycles utilisés en routine : le cycle « standard » et le cycle «Da Vinci ». Toutes ont été conformes. La qualification de la performance par le fabricant a consisté en une vérification de l'homogénéité et de la répartition des températures en plaçant des sondes dans la cuve, en un contrôle du niveau d'énergie ultrasonique à l'aide d'une sonde de mesure ayant pour but de contrôler l'efficacité et la force de cavitation générée par les transducteurs et transmise aux instruments placés dans le panier, en un contrôle du dosage du détergent et en tests d'efficacité de nettoyage.

La qualification de la performance du nettoyage a été réalisée à l'aide de deux tests de salissures Tosi ${ }^{\circledR}$ de la société Amcor ${ }^{\circledR}$ et de quatre tests Tosi-Lumcheck® branchés sur le système d'injection lors d'un cycle «Da Vinci » (spécifique pour les pinces du robot Da 
Vinci) et de deux tests Tosi-Lumcheck® branchés sur le système d'injection lors d'un cycle «standard ». Après nettoyage, il n'y avait plus aucune trace de résidus.

La qualification du nettoyage aux ultrasons n'est régie par aucune réglementation normative ou opposable. Cependant, l'équipe de stérilisation a souhaité compléter la qualification des performances en réalisant des tests de salissures supplémentaires avant son utilisation au quotidien par le personnel du service. Il a été choisi de ne pas contrôler l'efficacité de la cavitation et le fonctionnement des transducteurs car l'appareil venait d'être qualifié par le fabricant.

L'objectif de cette étude était de mettre en place un protocole de qualification du bac à ultrasons et de vérifier la qualité du nettoyage.

\section{Matériel et méthodes}

En l'absence de norme définissant la qualification des bacs à ultrasons, une revue de la littérature a été réalisée afin d'établir un protocole de qualification.

Les tests réalisés ont été ceux proposés par l'équipe du CHRU de Lille [2], du Centre Hospitalier de Chambéry [3] et du dossier thématique «Ultrasons » publié en 2014 par l'Association Française de Stérilisation [4]. Ils sont inspirés des normes des laveurs désinfecteurs dont les contraintes sont similaires.

Le protocole retenu pour la qualification du nouveau matériel est divisé en deux parties correspondant aux deux cycles utilisés en routine : le cycle Da Vinci (spécifique pour les pinces du robot Da Vinci) et le cycle standard (pour les autres dispositifs médicaux).

La solution détergente enzymatique alcaline utilisée est celle recommandée par le fournisseur de l'appareil. Il s'agit du détergent $E D A+{ }^{\circledR}$ du laboratoire Medisafe utilisé à une concentration de $0,7 \%$. 


\subsection{Cycle Da Vinci}

\subsubsection{Protocole utilisé}

Tout d'abord, des pinces du robot Da Vinci (un crochet, deux porte-aiguilles, deux pinces fenêtrées, une pince pleine et une paire de ciseaux) ayant atteint leur limite d'usage (dix utilisations) ont été recouvertes de Soiltest ${ }^{\circledR}$ du laboratoire Phagogène, mélange pré-formulé de lipides et de protéines dont la préparation et l'application sont rapides et faciles, puis laissées au repos pendant deux heures (Figure 1).

Les pinces souillées par la préparation de Soiltest ${ }^{\circledR}$ n’ont été ni brossées ni pré-désinfectées avant la mise en bac à ultrasons.

Parallèlement, des tests de salissures STF Load Check ${ }^{\circledR}$, distribués par le laboratoire Phagogène, mélange de deux sources de protéines, lipides et polysaccharides, ont par ailleurs été positionnés à différents endroits du bac lors du nettoyage des pinces robot.

Le test Tosi-Lumcheck® du laboratoire Amcor a également été utilisé. Le test de salissures, préparation à base de protéines dénaturées déposée sur une lame inox, a été inséré à l'intérieur d'un instrument témoin, se présentant sous la forme d'un tube creux et reproduisant les contraintes de nettoyage de ces dispositifs. Cet accessoire a ensuite été connecté au système d'irrigation du bac à ultrasons (Figure 2).

Les pinces robot, le test STF Load Check® et le test Tosi-Lumcheck® ont été soumis au cycle «Da Vinci », cycle programmé par le fournisseur, spécifique aux pinces du robot Da Vinci, d'une durée de 75 minutes. Il comprend un prélavage, un nettoyage de 20 minutes, une désinfection thermique de trois minutes et un rinçage final.

Enfin, le Résitest ${ }^{\circledR}$ a été employé après chaque cycle comportant des pinces robot afin de vérifier l'absence de protéines résiduelles après nettoyage. Il consiste à essuyer les 
instruments après nettoyage, à l'aide d'un écouvillon humidifié à l'eau. L'écouvillon est ensuite placé dans une solution test de détection des protéines résiduelles.

\subsubsection{Avantages des tests utilisés}

Le Soiltest ${ }^{\circledR}$ présente pour avantages de vérifier la qualité du procédé de nettoyage de tout type d'instrument et de pouvoir être appliqué aux endroits les plus difficiles d'accès grâce au pinceau fourni avec le produit.

Le test STF Load Check® a été choisi par l'équipe de stérilisation en raison de ses quatre faces et du support grillagé permettant de reproduire les zones d'ombre sur les instruments et la difficulté pour l'eau à atteindre l'ensemble des surfaces. Il permet donc de valider une efficacité de nettoyage multidirectionnelle.

Le test Tosi-Lumcheck®a été utilisé afin de contrôler la qualité du lavage des instruments creux.

Le Résitest ${ }^{\circledR}$ est un test simple à réaliser, rapide et sans incubation.

\subsubsection{Interprétation des résultats des tests}

Le test Soiltest ${ }^{\circledR}$ est conforme en l'absence de résidus de salissures après nettoyage. L'observation de résidus de salissures de couleur rouge indique que les dispositifs n'ont pas été correctement nettoyés.

S'agissant du test STF Load Check®, le lavage est conforme si l'indicateur a complètement viré à la fin du cycle. En revanche, la présence de traces rouges après nettoyage indique que le test n'est pas conforme et que le nettoyage n'est pas optimal.

Le Tosi-Lumcheck® est conforme si le test est indemne de salissures, après dévissage des deux parties de l'instrument témoin. 
Le Résitest ${ }^{\circledR}$ réagit avec les acides aminés et les peptides des résidus de protéines présents et vire, au bout de dix secondes, vers une couleur grise à bleue si des protéines résiduelles sont présentes. Le résultat du Résitest® est conforme si le test ne change pas de couleur et reste dans les teintes rouges-orangées : dans ce cas, on peut affirmer qu'il n'y a pas de protéines résiduelles.

\subsection{Cycle standard}

Des séries supplémentaires comportant uniquement les tests de salissures STF Load Check® et le Tosi-Lumcheck®, sans instruments, ont été effectuées selon le cycle «standard». Ce cycle présente une durée réduite par rapport au cycle «Da Vinci » : 50 minutes au lieu de 75 minutes. Ce cycle comprend un prélavage (rinçage), un nettoyage de 20 minutes et un rinçage final sans phase de désinfection thermique.

\section{Résultats}

$\mathrm{Au}$ total, 17 séries de sept pinces ont été enduites de Soil Test® soit 119 essais. Les emplacements des pinces ont varié à chaque cycle (avant, milieu et arrière bac). Quatre à cinq STF Load Check® par série ont été répartis uniformément dans le bac.

Enfin, deux Tosi-Lumcheck ${ }^{\circledR}$ ont été connectés de façon diamétralement opposée pour chaque cycle (Figure3).

Sur 119 pinces robots nettoyées en bac à ultrasons, 32 ont été retrouvées sales en sortie de lavage. Cela correspond à un pourcentage de non-conformités égal à 26,8\%. Seulement trois cycles sur 32 ont été conformes (9\%), c'est-à-dire que toutes les pinces étaient propres après nettoyage.

Quarante-six pour cent des pinces les plus souvent non propres ont été les porte-aiguilles (15 pinces), suivis des pinces fenêtrées (14 pinces soit 44\%). Des traces de salissures ont été 
présentes dans deux cas pour la pince non fenêtrée (6\%) et dans un cas pour le crochet $(3 \%)$. Les ciseaux ont toujours été retrouvés propres en sortie de nettoyage (Figure 4).

Les endroits présentant le plus de salissures après nettoyage étaient les mors et les articulations des pinces (Figure 5).

Dans $100 \%$ des cas de pinces visuellement non propres après nettoyage, le Résitest ${ }^{\circledR}$ a viré vers une teinte grise ou bleue, c'est-à-dire qu'il restait des protéines du Soiltest@ sur les pinces après nettoyage. De la même façon, ce dernier est resté négatif (pas de changement de couleur) dans $100 \%$ des cas lorsque les pinces étaient visuellement propres après nettoyage.

Sur les 78 STF Load Check® répartis dans chaque cycle «Da Vinci », huit n'ont pas viré uniformément (10\%). Sur les sept séries supplémentaires réalisées en cycle «standard », un seul test sur 35 n'a pas viré correctement (3\%).

Les deux Tosi-Lumcheck® de chaque panier ont viré dans $100 \%$ des cas en cycle «Da Vinci » (17 cycles soit 34 tests) et en cycle «standard » (7 cycles soit 14 tests).

Les résultats de ces tests sont détaillés par cycle dans le Tableau I.

\section{Discussion}

Seules les pinces robot ont été testées car ce sont les dispositifs les plus fréquemment pris en charge dans le service dans le cadre du nettoyage aux ultrasons. Ce sont également les plus complexes à nettoyer. De plus, les pinces robot étant limitées à dix utilisations, les essais ont pu être réalisés sur des pinces ne pouvant plus être utilisées chez le patient mais toujours fonctionnelles, contrairement aux autres dispositifs nettoyés aux ultrasons qui n'ont pas cette utilisation limitée. Il n'était, par ailleurs, pas possible d'immobiliser des dispositifs fonctionnels de routine chirurgicale. 
Le taux de non-conformités après nettoyage aux ultrasons est élevé contrairement aux résultats de l'étude menée par Y. Touil et al. où $95 \%$ des instruments imprégnés de Soiltest ${ }^{\circledR}$ ont été retrouvés propres après un nettoyage de 10 min et $100 \%$ à 15 minutes [5]. Cependant, les pinces robot arrivent en théorie en stérilisation préalablement pré-désinfectées. Ainsi, les tests réalisés dans cette étude ne reflètent pas entièrement la pratique quotidienne ; ils ont été faits dans des conditions extrêmes : les instruments n'ont été ni pré-désinfectés ni brossés et ont séché pendant deux heures. Néanmoins, il n'est pas rare que des salissures visibles à l'œil nu soient encore présentes à l'arrivée en stérilisation.

Les non-conformités concernant les porte-aiguilles et les pinces bipolaires fenêtrées (91\%) sont peut-être liées à la configuration des pinces. En effet, la pince fenêtrée et le porte-aiguille présentent beaucoup d'aspérités. Ceci conduit à un nettoyage plus difficile, contrairement au crochet dont l'espace entre le corps et la tête de la pince est plus grand et donc plus accessible au nettoyage. Les ciseaux, quant à eux, présentent un accès difficile empêchant la fixation des salissures (Figure 6).

Ce fort taux de non-conformités après nettoyage seul aux ultrasons nous a permis d'insister auprès du personnel qu'un brossage-écouvillonnage reste essentiel pour garantir la propreté du matériel en sortie de bac à ultrasons.

Il n'a pas été trouvé d'explication quant au non virage de certains STF Load Check®. Trois séries de quatre tests supplémentaires ont été réalisées. Le cycle a été arrêté manuellement après la phase de prélavage, c'est-à-dire après trois minutes de rinçage à l'eau uniquement et après remplissage de la cuve avec du détergent. Sur les douze tests, tous étaient partiellement ou entièrement virés au bout des trois minutes alors que le cycle n'en était qu'à son démarrage. 
Cela nous amène à la même conclusion que $\mathrm{C}$. Fayard concernant ces tests : ils semblent peu reproductibles et non adaptés à l'évaluation du nettoyage en bac à ultrasons, peut-être en raison de leur support souple qui nous avait préalablement séduits [3].

\section{Conclusion}

La qualité du nettoyage avec le bac à ultrasons SI PCF Medisafe est sans aucun doute améliorée par rapport à l'ancien bac à ultrasons notamment grâce à l'irrigation et à la désinfection thermique mais au détriment d'un cycle plus long (minimum 50 minutes contre 15 minutes auparavant). De plus, le cycle en bac à ultrasons reste toujours suivi d'une procédure manuelle de séchage.

Les résultats de ces tests ont été présentés au personnel lors de la formation à l'utilisation de l'appareil afin de leur faire comprendre la nécessité, avant la mise en bac à ultrasons, de brosser et d'écouvillonner les instruments afin de faciliter le nettoyage. Ceci est particulièrement important pour la partie utile des instruments de chirurgie robotique, notamment les porte-aiguilles et les pinces fenêtrées.

Un contrôle régulier de l'efficacité du nettoyage par des tests de salissures semble être intéressant à mettre en place. Le Tosi-Lumcheck ${ }^{\circledR}$ sera privilégié afin de vérifier la qualité du nettoyage intérieur car la plupart des instruments pris en charge en bac à ultrasons au sein du service sont des corps creux. Il sera associé à un autre test de salissure, sur un support dur comme le Tosi ${ }^{\circledR}$ ou le Pure-Check A®. Une utilisation uniquement ponctuelle du Soil test ${ }^{\circledR}$ peut être envisagée car la procédure est plus contraignante : dix minutes sont nécessaires pour enduire les instruments, suivies de 30 minutes minimum de séchage et de 75 minutes de cycle. De plus, les conditions de stockage de la solution de Soil test ${ }^{\circledR}$ avant et après reconstitution sont contraignantes (au réfrigérateur à $5^{\circ} \mathrm{C}$ ) rendant sa mise en œuvre peu aisée. 


\section{$\underline{\text { Figures }}$}

Figure 1 : Pince robot enduite de Soiltest ${ }^{\circledR}$

Figure 1: Robotic pliers tarnished with Soiltest ${ }^{\circledR}$

Figure 2 : Instrument témoin où est inséré le test de salissure

Figure 2 : Control device where the soilingis inserted

Figure 3 : Mise en place des pinces robot et des tests de salissures dans le bac à ultrasons

Figure 3: Positioning the robot pliers and soiling tests in the ultrasonic tub

Figure 4 : Pourcentage de non-conformités par type de pinces robot

Figure 4: Non-compliance percentage by robotic pliers type

Figure 5 : Porte-aiguille et pince fenêtrée présentant encore des traces de salissures au niveau des mors après nettoyage

Figure 5: Needle driver and fenestrated forceps still showing signs of dirt on the jaws after cleaning

Figure 6 : Porte-aiguille, crochet et ciseaux

Figure 6: Needle driver, cautery hook and scissors

\section{Tableau}

Tableau I : Résultats des tests effectués

Table I: Results of tests 


\section{Bibliographie}

[1] Denis C. Prise en charge des DMR du robot DA VINCI. 34èmes Journées nationales d'études sur la stérilisation dans les établissements de santé, Lyon, avril 2012. Disponible sur : http://www.cefh-ceps.com/actualite/ateliers_2012/C\%20DENIS.pdf (accès en date du 8 décembre 2018).

[2] Renaud A, Denis C. Comment qualifier un irrigateur ultrasonique ? 34èmes Journées nationales d'études sur la stérilisation dans les établissements de santé, Lyon, avril 2012. Disponible sur : http://www.cefhceps.com/actualite/posters/34ieme_journee/Comment\%20qualifier\%20un\%20irrigateur\%20ul trasonique\%20v00-2[1].pdf (accès en date du 8 décembre 2018).

[3] Fayard C. Méthode de contrôle des performances des bacs ultrasons. $2^{\text {èmes }}$ Journées internationales francophones de stérilisation, Marrakech, septembre 2014. Disponible sur: https://www.aster-info.be/wp-content/uploads/2014/11/07.-M\%C3\%A9thode-decontr\%C3\%B4le-des-performances-des-bacs-ultrasons-Camille-Fayard.pdf (accès en date du 8 décembre 2018).

[4] Association française de stérilisation. Dossier thématique «Les ultrasons », octobre 2014. Disponible sur : https://www.sf2s-sterilisation.fr/wp-content/uploads/2016/08/Ultrasons\%20\%2026\%2009\%202014.pdf (accès en date du 8 décembre 2018).

[5] Touil Y, Soumaré K, Rolland N et al. Qualification et contrôle de bacs à ultrasons en stérilisation. Pharm Hosp Clin $2016 ; 51: 88-9$. 


$$
16
$$




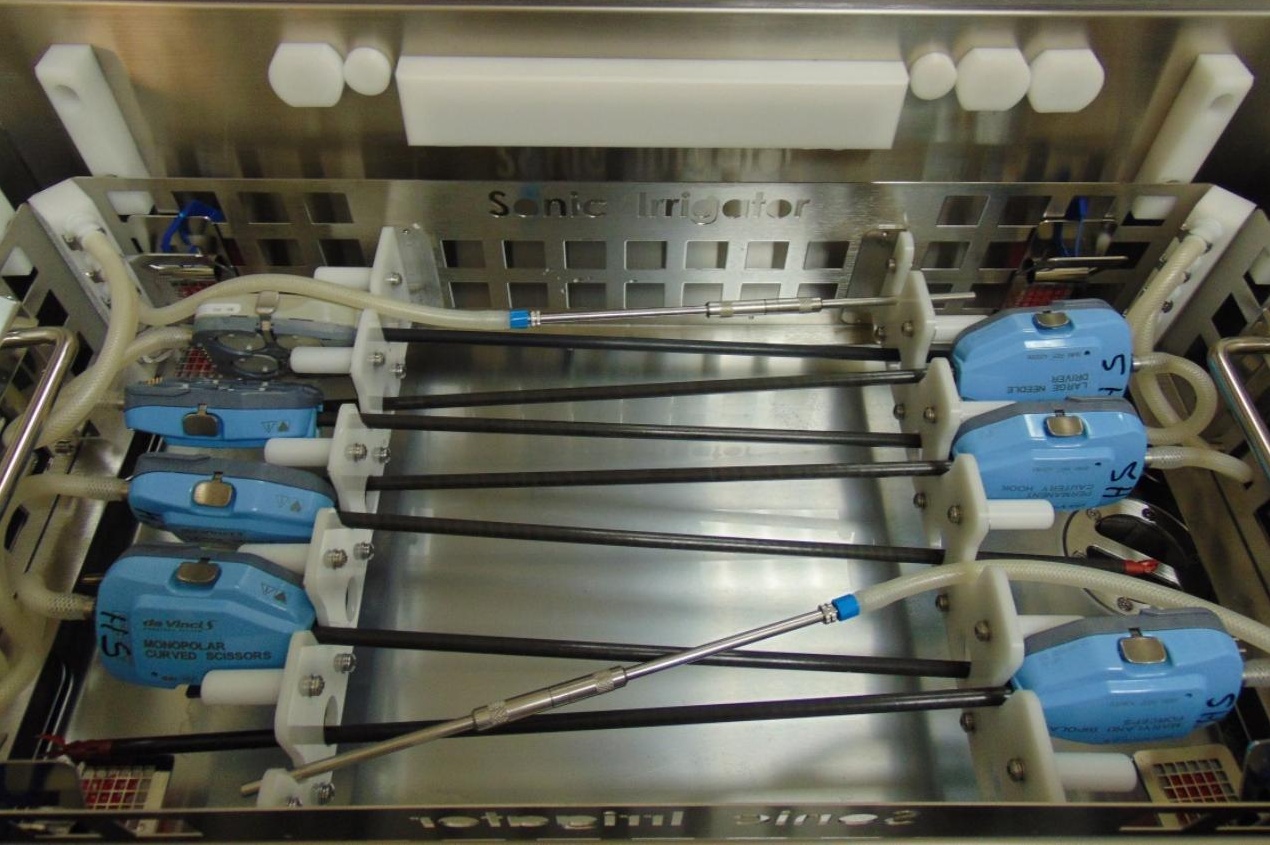




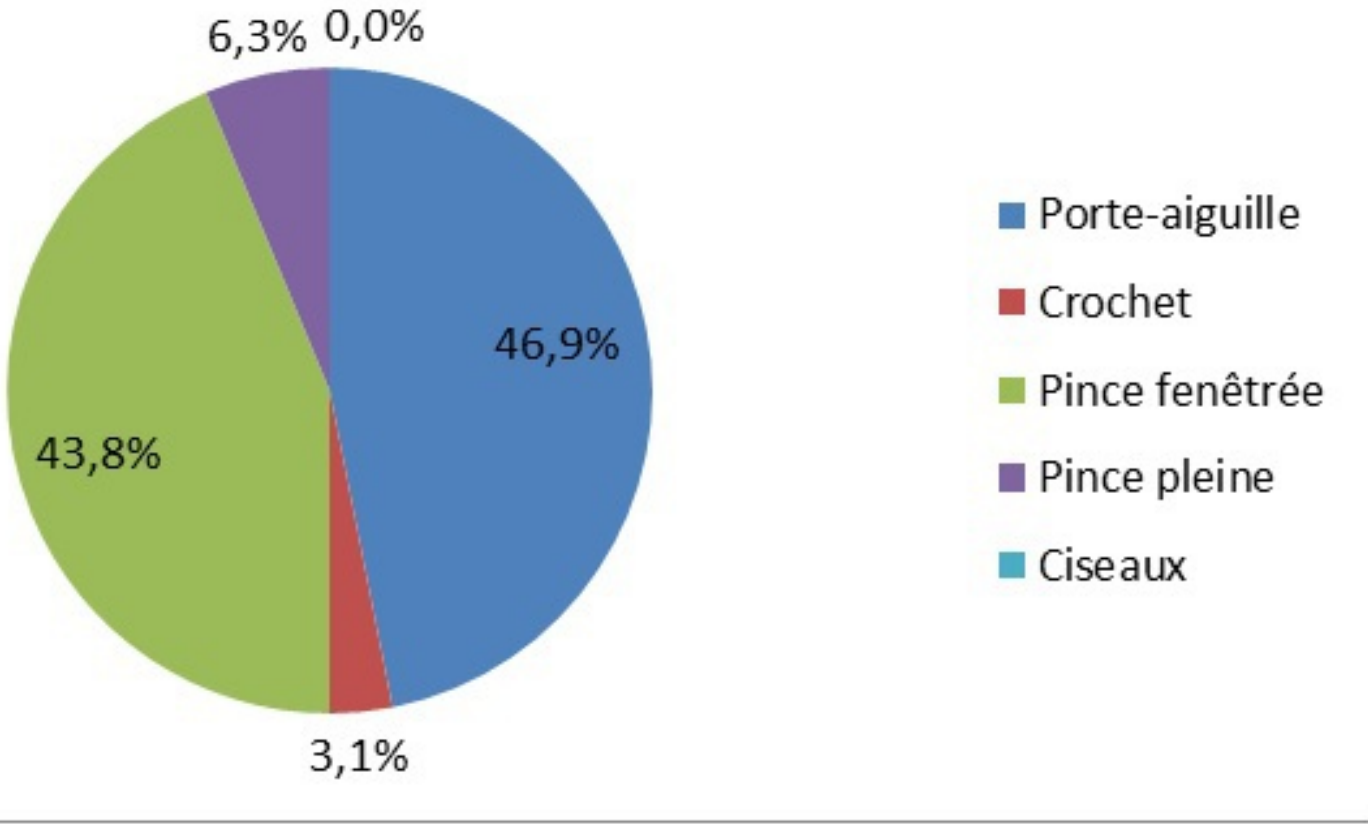


Tableau I : Résultats des tests effectués (Table I : Results of tests)

\begin{tabular}{|c|c|c|c|c|c|c|}
\hline Série & $\begin{array}{l}\text { Nombre total de pinces } \\
\text { dans le cycle }\end{array}$ & $\begin{array}{c}\text { Nombre de pinces non } \\
\text { propres après } \\
\text { nettoyage }\end{array}$ & $\begin{array}{c}\text { Type de pinces non propres } \\
\text { après nettoyage }\end{array}$ & $\begin{array}{c}\text { Tosi- } \\
\text { Lumcheck }^{\circledR}\end{array}$ & STF Load Check ${ }^{\circledR}$ & RésiTEST $^{\circledR}$ \\
\hline Da Vinci 1 & 7 & 2 & Porte-aiguille & Conforme & Non conforme & Positif \\
\hline Da Vinci 2 & 7 & 2 & Porte-aiguille & Conforme & Conforme & Positif \\
\hline Da Vinci 3 & 7 & 1 & Porte-aiguille & Conforme & Non conforme & Positif \\
\hline Da Vinci 4 & 7 & 1 & Porte-aiguille & Conforme & Conforme & Positif \\
\hline Da Vinci 5 & 7 & 1 & Crochet & Conforme & Conforme & Positif \\
\hline Da Vinci 6 & 7 & 0 & I & Conforme & Conforme & Négatif \\
\hline Da Vinci 7 & 7 & 0 & I & Conforme & Non conforme & Négatif \\
\hline Da Vinci 8 & 7 & 0 & I & Conforme & Conforme & Négatif \\
\hline Da Vinci 9 & 7 & 3 & $\begin{array}{l}\text { Porte-aiguille et pince } \\
\text { fenêtrée }\end{array}$ & Conforme & Conforme & Positif \\
\hline Da Vinci 10 & 7 & 3 & $\begin{array}{l}\text { Porte-aiguille et pince } \\
\text { fenêtrée }\end{array}$ & Conforme & Non conforme & Positif \\
\hline Da Vinci 11 & 7 & 4 & $\begin{array}{l}\text { Porte-aiguille, pince } \\
\text { fenêtrée, pince pleine }\end{array}$ & Conforme & Conforme & Positif \\
\hline Da Vinci 12 & 7 & 3 & $\begin{array}{l}\text { Porte-aiguille et pince } \\
\text { fenêtrée }\end{array}$ & Conforme & Conforme & Positif \\
\hline Da Vinci 13 & 7 & 4 & $\begin{array}{l}\text { Porte-aiguille et pince } \\
\text { fenêtrée }\end{array}$ & Conforme & Conforme & Positif \\
\hline Da Vinci 14 & 7 & 5 & $\begin{array}{l}\text { Porte-aiguille, pince } \\
\text { fenêtrée, pince pleine }\end{array}$ & Conforme & Non conforme & Positif \\
\hline Da Vinci 15 & 7 & 1 & Pince fenêtrée & Conforme & Non conforme & Positif \\
\hline Da Vinci 16 & 7 & 1 & Porte-aiguille & Conforme & Conforme & Positif \\
\hline Da Vinci 17 & 7 & 1 & Porte-aiguille & Conforme & Non conforme & Positif \\
\hline Standard 1 & I & 1 & 1 & Conforme & Conforme & 1 \\
\hline Standard 2 & I & I & I & Conforme & Conforme & I \\
\hline Standard 3 & I & I & I & Conforme & Conforme & 1 \\
\hline Standard 4 & I & I & 1 & Conforme & Non conforme & 1 \\
\hline Standard 5 & 1 & I & 1 & Conforme & Conforme & I \\
\hline Standard 6 & I & I & I & Conforme & Conforme & I \\
\hline Standard 7 & I & I & I & Conforme & Conforme & I \\
\hline
\end{tabular}

